\title{
A Feedback Based Adaptive Marking Algorithm for Assured Service
}

\author{
Fanjun Su, Chunxue Wu, and Guoqiang Sun \\ College of Computer Engineering, University of Shanghai for Science and Technology, \\ Shanghai 200093, China \\ sufanjun@163.com, sufanjun@usst.edu.cn
}

\begin{abstract}
We propose an algorithm to realize proportional bandwidth allocation in DiffServ networks. According to the feedback information, ingress routers adjust Committed Information Rates (CIRs) of different aggregates using AIMD way to let the network reach an exactly subscribed state. Based on CIRs, packets are marked. Simulations with ns2 show that proportional bandwidth allocation under different network conditions can be achieved.
\end{abstract}

Keywords: DiffServ, Proportional bandwidth allocation, AIMD.

\section{Introduction}

To provide assured service in DiffServ [1] networks, the markers at ingress routers (e.g. srTCM [2]) mark packets that obey the profile to high priority (e.g. IN), and mark packets that beyond the profile to low priority (e.g. OUT). The queue management mechanism operated at the core routers (e.g. RIO [3]) will preferentially treat high priority packets.

Ideally, proportional bandwidth allocation should be realized between different aggregates. Suppose a bottleneck link whose capacity is $C$, which is shared by $N$ aggregates. Let $C I R_{i}$ denote Committed Information Rate (CIR) of aggregate $i$, and $R_{i}$ denote the allocated bandwidth, where $1<=i<=N$. Then we say a network is undersubscribed when $\sum_{i} C I R_{i}<C$, over-subscribed when $\sum_{i} C I R_{i}>C$, and exactly subscribed when $\sum_{i} C I R_{i}=C$. If $R_{i}=C I R_{i} * \mathrm{C} / \sum_{j} C I R_{j}$, we say it is proportional bandwidth allocation. However, theoretic and simulation research in $[4,5,6]$ reveal that in DiffServ networks bandwidth are allocated unfairly between different aggregates unless the network is exactly subscribed. Hongjun $\mathrm{Su}$ [7] proposed an improved time sliding window based three color marker, but this algorithm can get better fairness only for low to middle level of provision. The proposal in [6] needs the modification of TCP in senders and receivers, and requires the support of ECN mechanism. When different aggregates start at different time, it cannot work well.

Therefore, in this paper a new algorithm named Feedback Based Adaptive Marking algorithm (FBAM) is proposed to achieve proportional bandwidth allocation between different aggregates. 


\section{Feedback Based Adaptive Marking Algorithm}

FBAM algorithm includes three parts:

(1) Subscription information feedback mechanism

Non-overlapped RIO [3] is wildly used in core routers. The calculation of $q_{\text {out }}$ includes IN and OUT packets, while the calculation of $q_{i n}$ only includes IN packets. Therefore, only after all OUT packets have been dropped does IN packets begin to be dropped.

We set a variable $q_{c o n g}$, which corresponds to an exactly subscribed state. When $q_{\text {in }}$ $<q_{c o n g}$, it means that the network is in an under-subscribed state. When $q_{\text {in }}>q_{c o n g}$, the network is in an over-subscribed state. According to the mechanism of RIO, we set $q_{\text {cong }} \cong q_{\text {in }}^{\text {min }}$. Feedback module monitors the value of $q_{i n}$. When $q_{i n}>q_{\text {cong }}$, feedback module will send a two-value feedback information to the ingress routers. We choose one unused bit in the packet head, and we call it subscription-bit. When a packet arrives or leaves the DiffServ domain, edge routers set the value of the subscriptionbit to 0 . When detecting $q_{\text {in }}>q_{\text {cong }}$, feedback module located in core routers will set the value of the subscription-bit to 1 .

To fully utilize the resource of the network, we use the reverse forward packets to transfer such feedback information. For DiffServ network, the topology of core networks usually is simple, so a static configuration can be set. In addition, most of the traffic in the Internet is based on TCP, so in most cases, there exists reverse ACK packets which can be used to transfer feedback information.

(2) Adjustment of CIRs at Ingress Routers

When a packet coming from core routers arrives, ingress routers will read the value of subscription-bit in the packet head. If the value of the subscription-bit is 0 , ingress routers increase CIRs of the aggregates at an interval $\delta$ as follows:

$$
C I R_{i, t}=C I R_{i, t-1}+\alpha * C I R_{i, 0}
$$

Where $C I R_{i, 0}$ is the original CIR of aggregate flow $i$ and $C I R_{i, t}$ is the CIR of aggregate $i$ at time $t$ after adjustment, and $\alpha$ is the increment factor.

If the value of subscription-bit is 1 , the ingress routers decrease CIRs as follows:

$$
C I R_{i, t}=(1-\beta) C I R_{i, t-1}
$$

Where $\beta$ is the decrement factor. Therefore, we can find the mechanism we mentioned has an idea of AIMD (additive increase multiplicative decrease). The convergence, stability, and fairness of AIMD have been analyzed in [8]. It should be emphasized that different ingress routers implement AIMD adjustment independently without the communication between them.

(3) Packet marking

We can use a rate meter such as TSW [2] to measure the sending rate of aggregate flows. Let $a v g \_r a t e_{i}$ denotes the average rate of aggregate flow $i$. When $a v g_{-}$rate $_{i}<=$ $C I R_{i}$, all packets are marked as IN. When avg_rate $_{i}>C I R_{i}$, let $P_{0}=C I R_{i} /$ avg_rate ${ }_{i}$, and $P_{1}=1-P_{0}$. Packets are marked as IN with probability $P_{0}$, and OUT with probability $P_{1}$. It can be inferred that such marking policy ensures that throughput of IN packets of the aggregate flow $i$ equals $C I R_{i}$ approximately. 


\section{Experimental Evaluations}

\section{(1) Simulation Topology and Configuration}

We adopt ns2 [9] as the simulation tool. As shown in Fig.1, IE1, IE2, IE3 are ingress edge routers, and EE1, EE2, EE3 are egress edge routers. R1 and R2 are core routers. $C_{1}$ is the bandwidth of the bottleneck link, and $C_{2}$ is the bandwidth of link IE3-R1. Every aggregate flow contains 9 TCP connections and 1 UDP connection respectively. The rate of UDP is $0.1 * C I R_{i, 0}$. The edge router queues employ drop-tail policy, and core router queues are managed by non-overlapping RIO algorithm. The parameters of

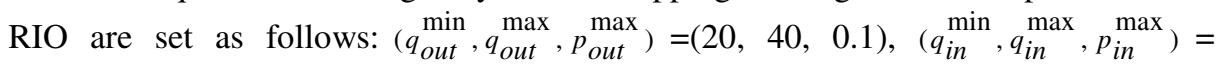
(40,80, 0.02), $q_{\text {cong }}=42$. The CIR adjustment parameters are set as follows: $\alpha=$ $0.002, \beta=0.002$, and $\delta=20 \mathrm{~ms}$. In the simulation, we use TSW [2] as rate meter. The Win_length of TSW is set to 0.1 second.

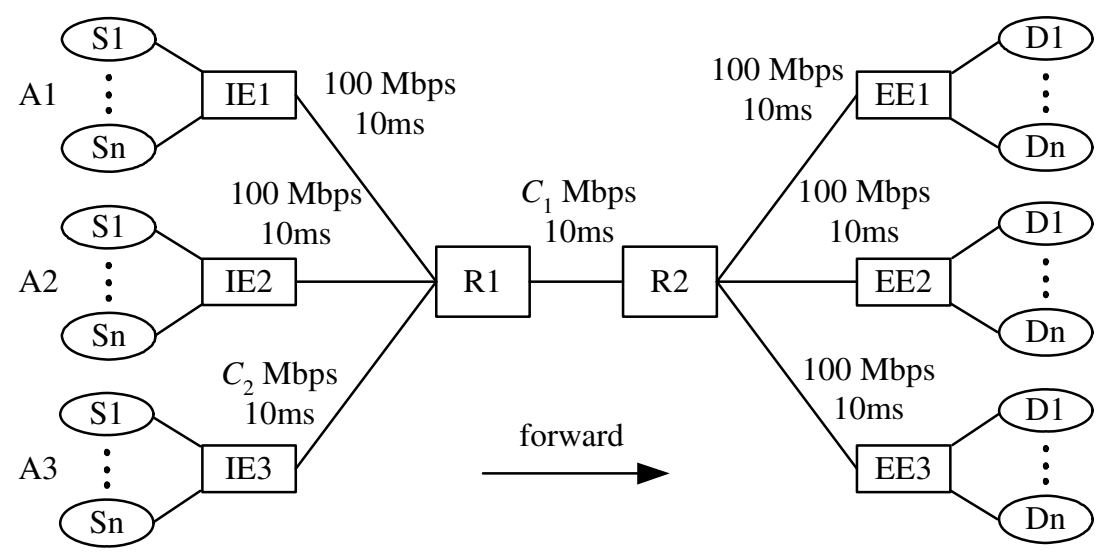

Fig. 1. Simulation topology and configuration

(2) Simulation results

Our first simulations are used to valid the effect of adjustment of CIR using AIMD way. Aggregates A1, A2, A 3 are used. We set $C_{2}=100 \mathrm{Mbps}$. The simulations are carried out in both the under-subscribed case and the over-subscribed case. Here we only list one result in over-subscribed case. $\left\{C I R_{i, 0}\right\}$ is set to $(2,5,10) \mathrm{Mbps}$, and $C_{1}=$ 10Mbps. Therefore, CIRs should be adjusted to $(1.18,2.94,5.88) \mathrm{Mbps}$, which corresponds to an exactly subscribed state. As shown in Fig. 2 (1), after adjustment, CIRs of different aggregates oscillate around ideal values, so proportional bandwidth allocation has been achieved as shown in Fig. 2 (2). In under-subscribed case, we get the same conclusions from the simulations.

We also carry out other simulations, such as the simulation in the case of aggregates start or close at different time and the case of aggregates have different bottleneck link. All the simulation results show that our algorithm can work well in different cases. 


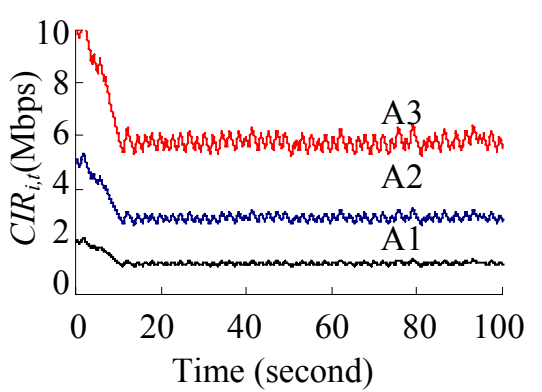

(1) CIR adjustment

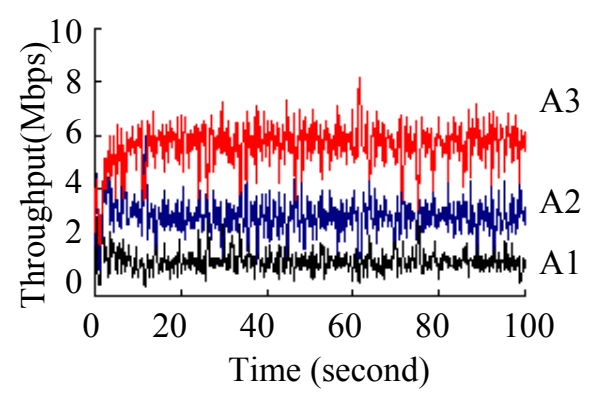

(2) Throughput

Fig. 2. Adjustment of CIRs and the bandwidth allocation in over-subscribed case

\section{Conclusions and the Future Work}

FBAM algorithm can detect the subscription state of the network, and send feedback information via reverse forward packets. According to the feedback information, ingress routers will adjust CIRs using AIMD way to reach an exactly subscripted state. Based on the adjusted CIR, FBAM marks the packets. Simulation results show that our algorithm can realize proportional bandwidth allocation under different network conditions. FBAM algorithm doesn't need communication between different ingress routers. Feedback information doesn't require special message packets. More simulations are needed for the optimal choice of parameters, such as $\alpha, \beta$.

\section{References}

1. Blake, S., Black, D., Carlson, M., et al: Architecture for differentiated services. RFC2475 (1998)

2. Heinanen, J., Guerin, R.: A single rate three color marker. IETF RFC2697 (1999)

3. Clark, D., Fang, W.: Explicit allocation of best effort packet delivery service. ACM Transactions on Networking (1998) 6(4): 362-373

4. Seddigh, N., Nandy, B., Pieda, P.: Study of TCP and UDP interaction for the AF PHB. http://www.watersprings.org/pub/id/draft-nsbnpp-diffserv-udptcpaf-00.txt (1999)

5. Seddigh, N., Nandy, B., Pieda, P.: Bandwidth assurance issues for TCP flows in a differentiated services network. Proceedings of Globecom, Rio De Janeiro (1999) 3: $1792-1798$

6. Park, E.C., Ho, C.: Proportional bandwidth allocation in DiffServ networks. IEEE INFOCOM (2004)

7. Su, H., Atiquzzaman, M.: ItswTCM: A new aggregate marker to improve fairness in DiffServ. IEEE GLOBECOM (2001) 25-29

8. Kelly, F., Maulloo, A., Tan, D.: Rate control for communication networks: shadow prices proportional fairness and stability. Journal of the Operational Research Society (1998) 49(3): $237-252$

9. Network simulator-ns2. http://www.isi.edu/nsnam/ns 